\title{
Structural Evolution of B-MCM-36 and B-ITQ-2 from B-MCM-22
}

\author{
Se-Young Kim, Soo-Hyun Jang, and Wha-Seung Ahn ${ }^{*}$ \\ Department of Chemical Engineering, Inha Universiry, Incheon 402-751, Korea. "E-mail: whasahn@inha.ac.kr \\ Received June 17, 2006
}

Key Words : Zeolite, B-MCM-22, B-MCM-36, B-ITQ-2

MCM-22 is a MWW type zeolite, which is made of $10-$ membered sinusoidal channels and 12-membered supercages, which are positioned next to each other independently in a three dimensional arrangement. This combination of large and medium pores was reported to be of great advantage in a variety of catalytic applications in petrochemical industry. ${ }^{1}$ Recently, several studies have been reported on isomorphous substitution of $\mathrm{Al}$ in MCM-22 with $\mathrm{B}, \mathrm{Fe}$, and $\mathrm{Ga}^{2-4}$ Among these, we have been particularly interested in B-MCM-22 which can be used as a starting material for Ticontaining MCM-22, better known as Ti-MWW. Ti-MWW demonstrated superior catalytic performances in selected liquid phase epoxidation reactions using $\mathrm{H}_{2} \mathrm{O}_{2}$ as an oxidant to that of the best known commercial catalyst, TS-1 (titanium silicalite-1). ${ }^{5}$

Direct isomorphous substitution of Ti for $\mathrm{Si}$ in MCM-22 structure is not possible and the MWW-type titanosilicate needs to be prepared using boric acid as structure-supporting agent by direct hydrothermal synthesis method. ${ }^{6}$ B-MCM22 , in this regard, is very useful because it can be prepared in alkali-free synthesis condition and possesses negligible acidity. Ti-MWW can also be prepared from a B-MCM-22 precursor via acid leaching of $\mathrm{B}$ species followed by postsynthetic $\mathrm{Ti}$ introduction and subsequent hydrothermal treatment. ${ }^{5}$

We are currently trying to prepare Ti-MCM- 36 and TiITQ-2 with modified textual properties. MCM-36 is a pillared molecular sieve with mesoporous region generated by expansion of the MCM-22 inter-layers via swelling/ pillaring. ${ }^{7}$ ITQ-2, on the other hand, can be prepared by exfoliation of swollen MCM-22 by sonication and the nanostructure obtained can afford reactants nearly unlimited access to the external zeolite-type catalytic sites. ${ }^{8}$ These features would be strongly desirable for handling larger and bulkier organic molecules in partial oxidation reactions such as in diesel fuel formulation. Obviously, B-MCM-36 and BITQ-2 need to be prepared from a B-MCM-22 precursor in order to prepare Ti-containing analogues.

In this paper, we report briefly the synthesis of B-MCM36 and B-ITQ-2 starting from a B-MCM-22 precursor. To the best of our knowledge, no synthesis on B-MCM-36 and B-ITQ-2 zeolites has been reported so far.

\section{Experimental Section}

B-MCM-22 was prepared following the synthesis method reported by Millini $e t a l^{2}$ at the molar ratio of the substrate mixture of $\mathrm{SiO}_{2}: 0.67 \mathrm{~B}_{2} \mathrm{O}_{3}: 1.4 \mathrm{PI}$ (piperidine) : $19 \mathrm{H}_{2} \mathrm{O}$.

B-MCM-36 was prepared similar to the synthesis method reported for Al-MCM-36 by $\mathrm{He}$ et al ${ }^{7}$ with the gel composition of B-MCM-22 (P) : 4 CTMACl : 1.2 TPAOH. Here, $\mathrm{P}$ designates a precursor phase without calcination, and CTMACl and TPAOH represent cetyltrimethylammonium chloride and tetrapropylammonium hydroxide, respectively. The mixture was refluxed at $373 \mathrm{~K}$ for $68 \mathrm{~h}$ and was aged at room temperature for $4 \mathrm{~h}$. For pillaring, the swollen material was mixed with tetraethylorthosilicate (TEOS, 98\%, Aldrich) at a weight ratio of $1: \mathrm{x}$, where $\mathrm{x}$ was varied between 1 and 5. The mixture was heated at $363 \mathrm{~K}$ for $25 \mathrm{~h}$ in nitrogen under stirring. Subsequently, hydrolysis step was carried out at $413 \mathrm{~K}$ for $6 \mathrm{~h}$. The product was calcined at $723 \mathrm{~K}$ for $3 \mathrm{~h}$ in nitrogen and at $812 \mathrm{~K}$ for $6 \mathrm{~h}$ in air (heating rate of $2 \mathrm{~K} /$ min).

B-ITQ-2 was synthesized based on the recipe for Al-ITQ2 by Corma $e t a l^{8}$ A swollen B-MCM-22 precursor was exfoliated using sonification $(100 \mathrm{~W}, 20 \mathrm{kHz})$ for $1 \mathrm{~h}$ at 323 $\mathrm{K}$ after $\mathrm{pH}$ of the mixture being adjusted to 12.5 . At the completion of the exfoliation process, $\mathrm{pH}$ of the mixture was adjusted to 2 by adding hydrochloric acid. The resulting material was centrifuged and calcined at $813 \mathrm{~K}$.

The crystallinity of the samples prepared was measured by $\mathrm{X}$-ray diffraction using Ni-filtered CuK $\alpha$ radiation (Rigakuminiflex) and the morphology of the samples was examined by SEM (Hitachi, S-4200). The specific surface areas and average pore diameter were determined by $\mathrm{N}_{2}$ adsorptiondesorption with the BET method at liquid nitrogen temperature using a Micromeretics ASAP 2000 automatic analyzer. The external surface areas were estimated by the $t$-plot method. The amount of $\mathrm{B}$ was determined by inductively coupled plasma emission spectrometry (ICP, JY-138).

\section{Results and Discussion}

The structural relationships between B-MCM-22 and its derivatives are schematically shown in Figure 1. B-MCM22 (P) has MWW lamellar structure with $10 \mathrm{MR}$ channels. After calcination, organic template molecules are removed and ordered linkages between the sheets formed, leading to a three-dimensional system. Swollen B-MCM-22 precursor is shown to have an expansion of interlayer distance and the pillared B-MCM-36 has a mesoporous region created by polymeric silica. B-ITQ-2 has a number of lamellar sheets as 


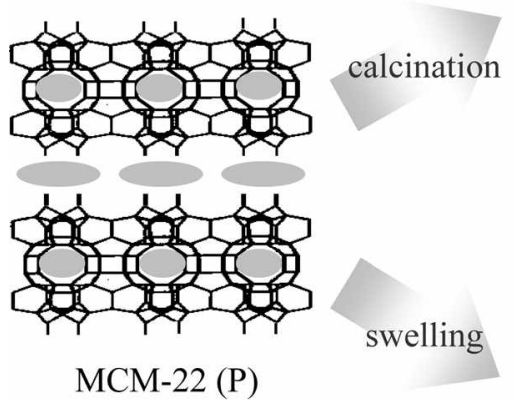

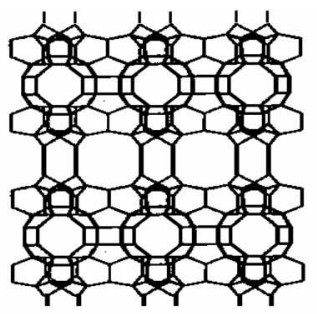

MCM-22
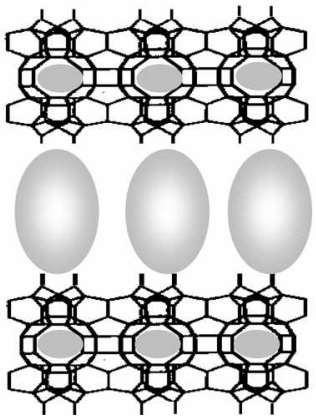

swollen material
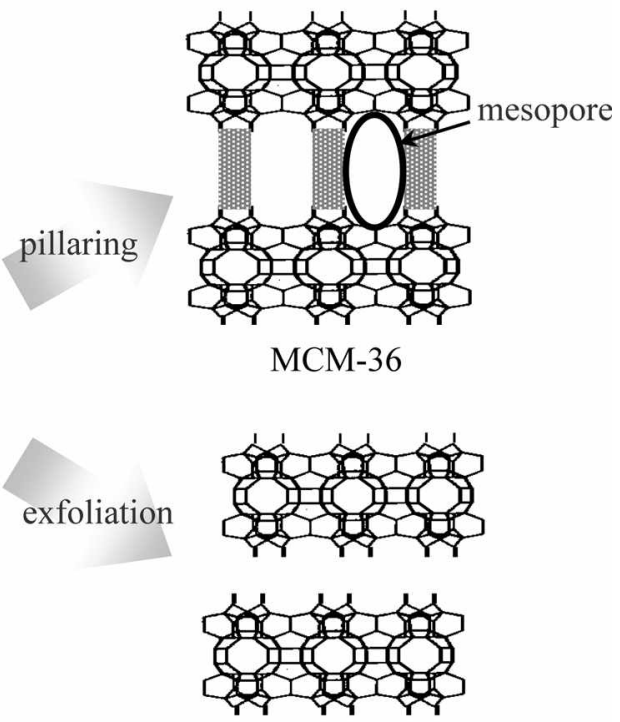

ITQ-2

Figure 1. Schematic representation of B-MCM-22, B-MCM-36 and B-ITQ-2.

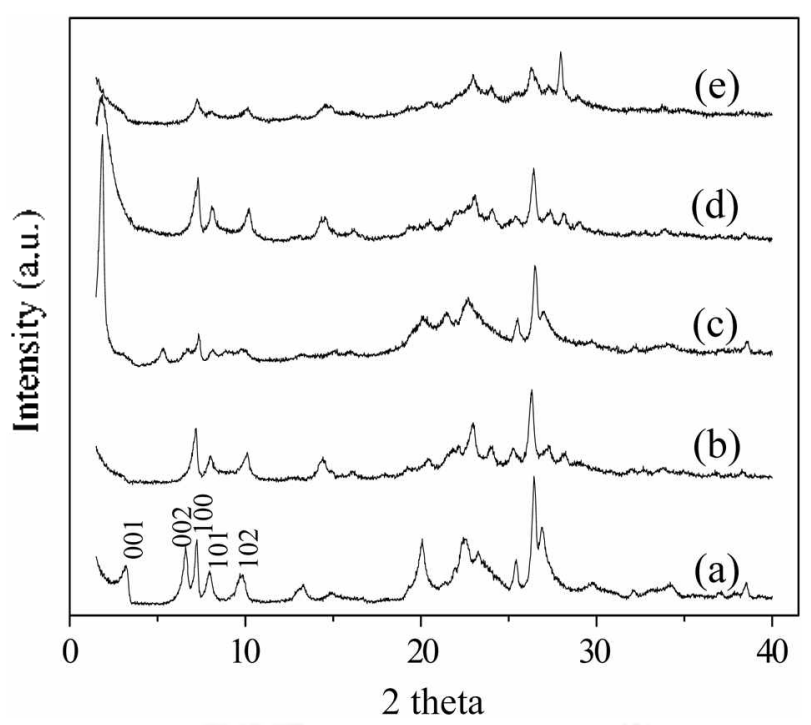

Figure 2. X-ray dintraction patterns: (a) as-synthesized B-MCM22, (b) calcined B-MCM-22 (calcined), (c) B-MCM-22 (swollen), (d) B-MCM-36, (e) B-ITQ-2.

a result of exfoliation. ${ }^{9}$

First of all, ICP analysis revealed that $\mathrm{Si} / \mathrm{B}$ ratio of the $\mathrm{B}$ MCM-22 product is only 33 , which was substantially less than the starting composition of the synthesis substrate mixture of $\mathrm{Si} / \mathrm{B}=3$. Thus only a small fraction of initial $\mathrm{B}$ was found incorporated into the zeolite structure. ${ }^{2}$

Figure 2 shows the XRD patterns of materials prepared. The as-synthesized B-MCM-22 shows the characteristic $(001)$ and $(002)$ peaks at $2 \theta=3-7^{\circ}$ due to c-axis. The $(002)$ peak at $2 \theta=6.6^{\circ}$ is shifted to $2 \theta=7-7.5^{\circ}$ after calcination. B-MCM-36 has a new peak at $2 \theta=1-2^{\circ}$ signaling the formation of mesopores, and B-ITQ-2 showed a decrease in peak intensities in general and demonstrated broaden peaks as a result of exfoliation due to size reduction. Exfoliation of B-ITQ-2 was, however, not achieved to the full extent judging by persisting peaks remained at $2 \theta=25-30^{\circ}$, part of which correspond to the $12 \mathrm{MR}$ supercages in the swollen structure. Extensive attempts to exfoliation by varying experimental condition in sonication time, power, temperature, and $\mathrm{pH}$ had all resulted in partial success only. It is not clear at the moment why the exfoliation did not proceed to full extent but it is noteworthy in that difficulties in exfoliation process has also been reported by several researchers earlier in Al-ITQ-2. ${ }^{8.10}$

Scanning electron micrograph images of B-MCM-22, BMCM-36, and B-ITQ-2 are shown in Figure 3. Morphology of B-MCM-22 indicates platelets and some large particles are aggregated together. B-MCM-36 had more highly agglomerated parts than B-MCM-22, and B-ITQ-2, despite of the $\mathrm{pH}$ adjustment, is also seemed to be made of platelets due to condensation of surface silanol groups after calcination.

Nitrogen adsorption isotherms of materials are compared in Figure 4. Whilst the isotherm of B-MCM-22 was type I due to the microporous nature of the material, B-MCM-36 showed type IV isotherm with a hysteresis loop at $\mathrm{p} / \mathrm{p}_{\mathrm{e}}=0.4$ for capillary condensation, which indicates the successful formation of mesopores after swelling and subsequent pillaring. ${ }^{7}$ Isotherm of B-ITQ-2 indicated that micropores are still maintained after the partial exfoliation process. Table I showed the BET surface areas and extemal surface areas of the samples prepared. The specific surface and extemal surface areas of B-MCM-36 and B-ITQ-2 were substantially higher than that of B-MCM-22. In B-MCM-36, pillaring causes an increase in external surface area, but $\mathrm{B}$ - 

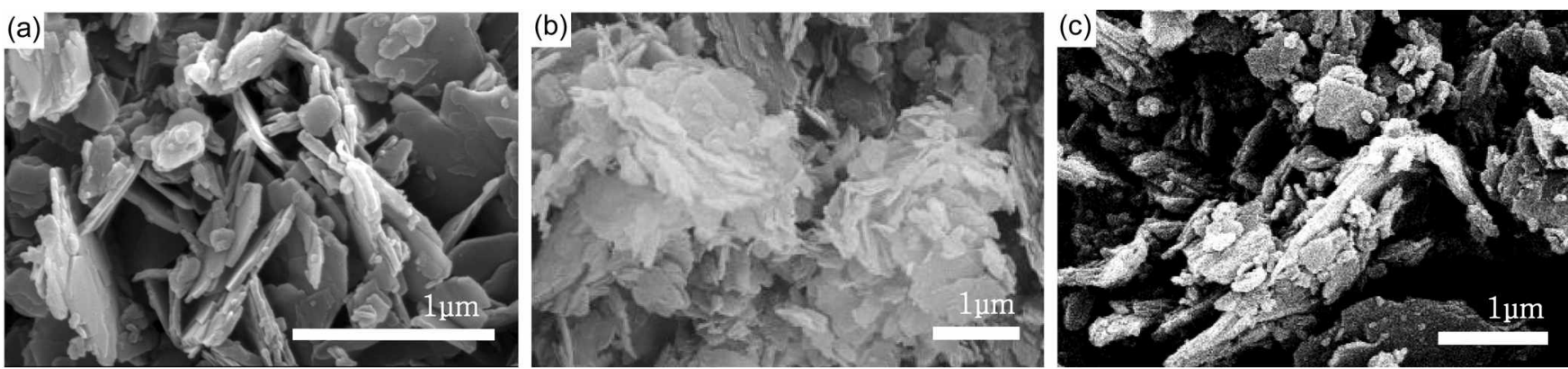

Figure 3. SEM images: (a) B-MCM-22, (b) B-MCM-36 and (c) B-ITQ-2.

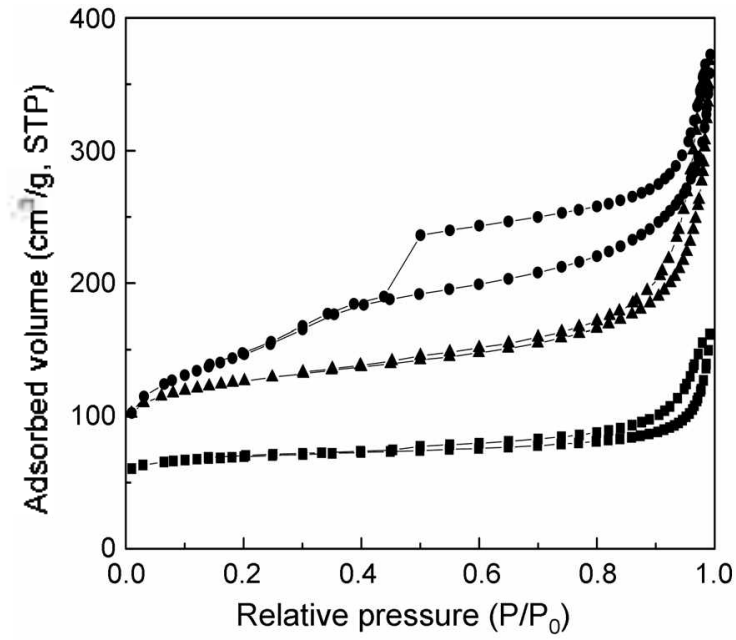

Figure 4. Nitrogen adsorption isotherms of B-MCM-22 ( $\square$ ), BMCM-36 ( • ), B-ITQ-2 ( $\boldsymbol{\Delta})$.

Table 1. Specific surface area and volume of samples

\begin{tabular}{cccc}
\hline Sample & $S_{\text {kon: }}\left(\mathrm{m}^{2} / \mathrm{g}\right)$ & $S_{\text {tat }}\left(\mathrm{m}^{2} / \mathrm{g}\right)$ & $V_{\text {total }}\left(\mathrm{cm}^{3} / \mathrm{g}\right)$ \\
\hline B-MCM-22 & 234 & 54 & 0.14 \\
B-MCM-36 & 520 & 113 & 0.52 \\
B-ITQ-2 & 432 & 161 & 0.40 \\
\hline
\end{tabular}

$V_{\text {lolal }}=$ total pore volume. $S_{\text {(total }}$ and $S_{\mathrm{exl}}=$ lotal and external surface areas.

ITQ-2 had shown a lower surface area than B-MCM-36 due to incomplete exfoliation as mentioned earlier. The extemal surface area of B-ITQ-2 was higher than that of B-MCM-36 to only a minor extent; had the exfoliation been successfully carried out, much higher increase in extemal surface area would have been measured. The pore volume of B-MCM-22 is only $0.14 \mathrm{~cm}^{3} / \mathrm{g}$, indicating that micropores are dominant. On the other hand, pore volume of B-MCM-36 with mesopores was $0.52 \mathrm{~cm}^{3} / \mathrm{g}$, whereas pore volume of B-ITQ-2 was $0.4 \mathrm{~cm}^{3} / \mathrm{g}$.

Polymeric silica pillars were introduced by TEOS hydrolysis at the relative weight ratio of TEOS to the swollen BMCM-22 of 5 to 1. However, excess amount of silica pillaring is apparently undesirable due to pore blocking over the interlayer region. Consequently, in order to minimize the amount of TEOS used without compromising the structural stability of B-MCM-36, we systematically varied the amount

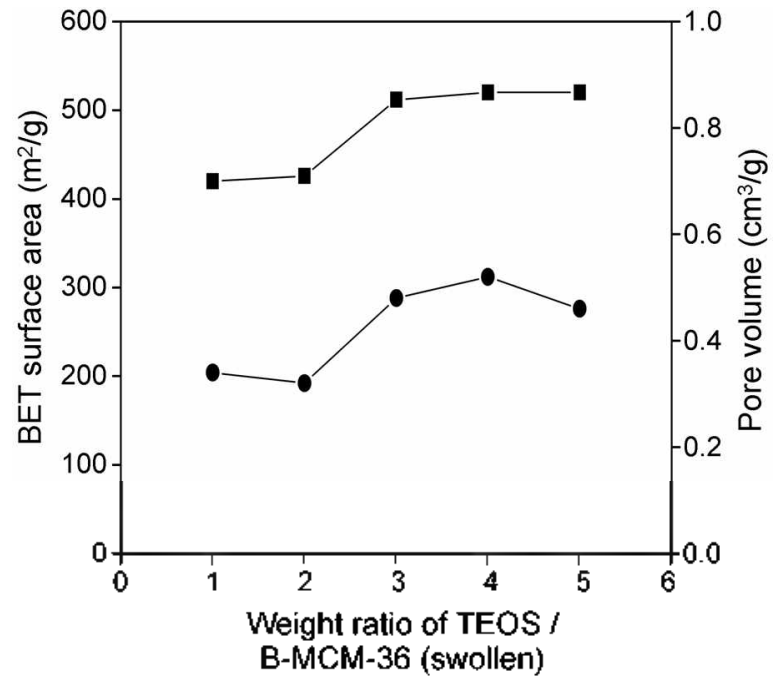

Figure 5. BET surface area ( $\boldsymbol{D}$ ) and pore volume ( $\bullet$ ) of BMCM-36 as a finction of TEOS amount introduced.

of pillaring agent TEOS from $5: 1$ to $1: 1$. The corresponding textual properties measured and the trend in the surface area and pore volume change following the pillaring is described in Figure 5. While BET surface area and pore volume of the sample with TEOS : swollen B-MCM-22 of 4 : 1 gave the best result, no apparent improvement in textual properties were indicated above the ratio of $3: 1$. This finding is expected to make a useful contribution to the synthesis of catalytically active Ti-MCM-36.

\section{Conclusion}

B-MCM-36 could be successfully prepared from a BMCM-22 precursor after swelling and pillaring process as confirmed by XRD analysis. Improved textural properties of substantially enhanced surface area accompanied by mesopores were obtained, which are desirable for handling relatively large organic molecules. The amount of pillaring agent TEOS to the $\mathrm{B}-\mathrm{MCM}-22$ (swollen) recommended was 3 : 1. Only partial success to B-ITQ-2 phase was achieved due to incomplete exfoliation.

Acknowledgment. This work was partly supported by Inha University research Grant (2006). 


\section{References}

1. Kennedy, G.; Lawton, S.; Rubin, M. J. An. Chen. Soc. 1994, 116. 11000 .

2. Millini, R.; Perego, G.; Parker, W; Bellussi, G; Carluccio, L. Micropor. Mater: 1995, 4, 221.

3. Testa, F.; Crea, F.; Diodati, G.; Pasqua, L.; Aiello, R.; Terwagne, G; Lentz. P.; Nagy, J. Micropor. Mesopor. Mater. 1999, 30, 187.

4. Morrison, R. A.; Rubin, M. K. U,S. Patent 5,382,742, 1995.
5. Wu, P.; Tatsumi, T. Chem. Commun. 2002, 10, 1026.

6. Wu, P.; Tatsumi, T.; Komatsu, T.; Yashima, T. J. Phss. Chem. B. 2001, $105,2897$.

7. He, Y.; Nivarthy, G; Eder, F.; Seshan, K.; Lercher, J. Micropor: Mesopor. Materials 1998, 25, 207.

8. Corma, A.; Fornes, V.; Pergher, S.; Maesen, T.; Buglass, J. Nature (Lond.) 1998, 396, 353.

9. Corma, A.; Forñes, V.; Martínez-Triguero, J.; Pergher, S. J. Catal. $1999,186,57$

10. Nuntasti, D.; Wu, P; Tatsumi, T. Chem. Lett. 2003, 32, 326. 